\title{
REFLEXÕES SOBRE AS DISFUNÇÕES SACROILÍACA E SEXUAL
}

\section{Tatiane Thomaz}

Pós-graduanda do curso de Fisioterapia Pélvica da Faculdade Inspirar, Campinas (SP), Brasil

\section{Erica Feio Carneiro Nunes}

Mestre em Ciências da Motricidade Humana; Docente da Universidade do Estado do Pará, Pará, Brasil.

E-mail: erica@perineo.net

\section{Gustavo Fernando Latorre}

Fisioterapeuta pélvico; Mestre em fisioterapia; Florianópolis, SC -Brasil
RESUMO: O objetivo deste estudo foi abordar a estudar relação entre disfunções sacroilíacas (DSI) e disfunção sexual (DS). Buscouse nas bases de dados SciELO e PUBMED por artigos de 2006 a 2012 nas línguas portuguesa e inglesa, com as palavras-chave articulação sacroilíaca e disfunção sexual feminina, resultando em sete artigos. Os resultados mostraram que fraturas da cintura pélvica estão relacionadas à DS feminina. Apesar de as bases teóricas apontarem clara relação entre outras DSI que não fraturas e a DS, esta relação não teve respaldo na literatura. Conclui-se que se necessitam de estudos explorando as alterações anatômicas e biomecânicas da articulação sacroilíaca, especialmente as que gerem fraqueza ou tensão da musculatura do assoalho pélvico como possíveis fatores etiológicos da DS.

PALAVRAS-CHAVE: Articulação Sacroilíaca; Disfunção sexual fisiológica; Mulheres.

\section{SACROILIAC AND SEXUAL DYSFUNCTIONS}

ABSTRACT: Relationships between sacroiliac (SID) and sexual dysfunctions (SD) are investigated by researching at SciELO and PUBMED databases for articles in English and Portuguese, published between 2006 and 2012, with keywords sacroiliac articulation and female sexual dysfunction. Seven articles were retrieved. Results showed that fractures of pelvic waist are related to female SD. Although theoretical bases indicate clear relationship among other non-fracture SIDs and SD, relationship was not found in the literature. Further studies should be undertaken on anatomical and biomechanical changes of the sacroiliac articulation, especially those that weaken or cause tension on the pelvic floor as possible etiological SD factors.

KEYWORDS: Sacroiliac joint; Sexual dysfunction, Physiological; Women.

\section{INTRODUÇÃO}

É cada vez mais reconhecida a importância da saúde sexual para a longevidade das relações afetivas e como parte da saúde global e bem-estar do indivíduo (LARA et al., 2008). Para a World Association for Sexual Health, a saúde sexual é um componente essencial da saúde e bem-estar, sendo um dos pilares da qualidade de vida nos seres humanos (GIRALDO et al., 2012). Especialmente com relação à população feminina, dificuldades de desempenho e satisfação sexual 
são problemas relevantes de saúde pública, acometendo grande parte da população e prejudicando sua qualidade de vida (HENTSCHEL et al., 2006; ABDO, 2009)

A definição de disfunção sexual feminina evoluiu, nos últimos 15 anos, refletindo o conceito de que o princípio da resposta sexual feminina é composto por uma variedade de domínios e categorias (JONES, 2002; ABDO, 2004). Atualmente, uma estandardização sobre o tema, baseada em evidências e efetuada por especialistas de cinco continentes, definiu que as disfunções sexuais femininas incluem distúrbios persistentes ou recorrentes de interesse/desejo sexual, distúrbios de excitação subjetiva e genital, transtorno do orgasmo, dor e dificuldade tanto na relação tentada ou concluída (BASSON et al., 2004).

No começo desta década foi realizada uma pesquisa com 2.835 indivíduos de ambos os gêneros, cuja finalidade foi estudar o comportamento sexual dos brasileiros. Dentre os resultados avaliados na população feminina, constatou-se que as principais queixas eram falta de desejo sexual, dificuldades de orgasmo e dor durante a relação sexual (ABDO et al., 2002).

Baseada em estudos anatômicos e fisiológicos, as alterações dos músculos do assoalho pélvico (MAP) estão entre as diversas causas das disfunções sexuais (MOREIRA; ARRUDA, 2010). O termo "músculos do assoalho pélvico" refere-se à camada muscular que fecha inferiormente a pelve óssea, tendo como funções básicas o suporte dos órgãos pélvicos, a manutenção da continência urinária e fecal, desempenhando ainda papel fundamental na função sexual feminina (FORTUNATO et al., 2006; PIASSAROLLI, 2010; BARACHO, 2012).

Em conjunto com outros músculos, os MAP são estabilizadores da cintura pélvica, coluna lombar e quadril, de forma que, uma postura equilibrada depende também do bom desempenho do assoalho pélvico e vice-versa, uma vez que os MAP se inserem em ossos do quadril, no qual outros músculos do corpo também, como os músculos do tronco e dos membros inferiores também se inserem, e assim, uma alteração postural pode alterar também a flexibilidade do assoalho pélvico, o que diminui sua eficiência, o que pode resultar em sintomas como urgência miccional, polaciúria e dispareunia (MIRANDA; SCHOR; GIRÃO, 2009).

Pode-se inferir também que a disfunção sacroilíaca poderia desencadear a disfunção dos MAP
(BARACHO, 2012). Uma alteração na região sacral pode levar a uma instabilidade e um desequilíbrio pélvico, o que possivelmente pode favorecer a fraqueza muscular, e assim as alterações no sacro podem acarretar comprometimento da força desses músculos (SOARES et al., 2011), podendo ser um agravante na disfunção sexual.

As articulações sacroilíacas são em parte uma articulação sinovial e em parte uma articulação sindesmodial, fixadas por fortes ligamentos, sendo responsáveis pela estabilidade da pelve e também por micromovimentos entre o sacro e os ílios. São envolvidas por potentes músculos que não tem nenhuma influência direta no movimento da articulação, mas podem interferir na mobilidade da mesma. A disfunção sacroilíaca pode ser caracterizada por anormalidades biomecânicas do posicionamento anatômico da articulação, como por exemplo, fixação, hipomobilidade, subluxação ou mau alinhamento, podendo ser unilateral ou bilateral (RIBEIRO; SCHMIDT; WURFF, 2003).

Os movimentos das articulações sacrilíacas e a sínfise púbica não possuem músculos que controlem diretamente os seus movimentos, eles são influenciados pela ação de músculos que movem a coluna lombar e os quadris, muitos dos quais se fixam no sacro e na pelve (MAGEE, 2005), exemplos são os músculos obturadores internos, piriforme, adutores, glúteos e abdominais, que são adjacentes aos MAP, desta forma, alterações em quaisquer um desses músculos podem ocasionar disfunções pélvicas devido ao desequilíbrio muscular, tensão e encurtamento dessas cadeias musculares ligadas à pelve (YENG et al., 2001; CALAIS-GERMAIN, 2005; MIRANDA; SCHOR; GIRÃO, 2009).

Além disso, as raízes L4 a $\$ 4$ são responsáveis pela inervação da articulação sacroílíaca e justificam a ampla distribuição da dor referida nas regiões sacral, pubiana e perineal, face posterior da coxa e da perna, face plantar do pé, além do espasmo reflexo do piriforme e músculos glúteos (YENG et al., 2001).Há sobreposição nervosa entre a inervação das articulações sacroilíacas e de todos os órgãos pélvicos, incluindo seu assoalho, via nervo pudendo, raízes S2, S3 e S4 (COSTA, 2013).

Considerando que a disfunção sexual feminina é uma doença de etiologia multicausal e ainda não 
totalmente esclarecida (BASSON et al., 2004), estudos que explorem possíveis novos fatores etiológicos são bem-vindos.

A possível relação entre a disfunção sacroilíaca, favorecendo a fraqueza da musculatura do assoalho pélvico que está intimamente ligada com a disfunção sexual feminina pode fomentar a hipótese de uma possível relação causal entre estas, podendoser mais um ponto de foco na avaliação física e culminar, posteriormente, no desenho de abordagens curativas e preventivas mais eficientes e eficazes. Desta forma, este estudo teve por objetivo estudara relação entre disfunções sacroilíacas e disfunção sexual

\section{METODOLOGIA}

Trata-se de uma revisão bibliográfica onde foram consultadas as bases de dados SciELO e PUBMED em busca de artigos que mencionassem simultaneamente as palavras-chave "articulação sacroilíaca" e "disfunção sexual feminina", bem como suas variantes em inglês. Foram incluídos estudos publicados de 2006 a 2012, usando os descritores nas línguas portuguesa e inglesa. Sendo excluídos artigos que unicamente citavam as palavras-chave, sem apontar qualquer tipo de relação entre as mesmas, ou que tratavam concomitantemente de outras patologias. Os artigos resultantes foram tabelados por autor, ano, e pela relação que apresentavam entre as duas variáveis estudadas.

\section{RESULTADOS}

Na base PubMed o cruzamento das duas palavraschave retornou um total de seis artigos, sendo quatro incluídos e dois excluídos por não estarem disponíveis na íntegra. Na base SciELO o cruzamento das expressões não retornou nenhum artigo, mas a utilização isolada da palavra-chave "sacriliac joint" retornou 15 artigos, dos quais 12 foram excluídos, particularmente por tratarem de técnicas para imaginologia, disfunções púbicas, discussão de técnicas cirúrgicas ou anestesiológicas. Os sete artigos restantes são resumidos no Quadro 1.

Ramirez e Lemus (2010) pesquisaram sobre

Quadro 1. Artigos encontrados nas bases pesquisadas

(continua)

\begin{tabular}{|c|c|c|}
\hline AUTOR & OBJETIVOS & RESULTADOS / CONCLUSÃO \\
\hline $\begin{array}{l}\text { Wright et al., } \\
2006\end{array}$ & $\begin{array}{l}\text { Estudar a prevalência de } \\
\text { disfunção sexual e excretora } \\
\text { em homens e mulheres após } \\
1 \text { ano de fratura pélvica. }\end{array}$ & $\begin{array}{l}21 \% \text { dos pacientes com fratura relataram disfunção sexual, contraposto a } 14 \% \text { dos } \\
\text { pacientes sem fratura e } 8 \% \text { versus } 4 \% \text { para disfunção excretora. Desses, destacou-se pior } \\
\text { resultados para lesões sacriliacas nos homens e diástase da sínfise púbica para mulheres. } \\
\text { Todos os pacientes com alguma disfunção apresentaram pior índice de qualidade de vida. }\end{array}$ \\
\hline $\begin{array}{l}\text { Mejía et al., } \\
2008\end{array}$ & $\begin{array}{l}\text { Estudar a anatomia, o } \\
\text { diagnóstico e os tratamentos } \\
\text { da dor sacrilíaca. }\end{array}$ & $\begin{array}{l}\text { A prevalência da dor sacroilíaca não é bem estudada, tendo inúmeras etiologias, podendo } \\
\text { ser simplificadas em causas extra ou intra-articular. O exame físico conta com } 12 \text { provas } \\
\text { provocativas, sugerindo diagnostico de disfunção sacroilíaca quando } 3 \text { dessas provas são } \\
\text { positivas. Exames de imagem também são importantes para o diagnostico. O tratamento } \\
\text { pode ser conservador, medicamentoso ou cirúrgico. }\end{array}$ \\
\hline $\begin{array}{l}\text { Cannada; } \\
\text { Barr, } 2010\end{array}$ & $\begin{array}{l}\text { Estudar a presença de } \\
\text { disfunção sexual em } \\
\text { mulheres após fratura } \\
\text { pélvica e sua interferência } \\
\text { no parto. }\end{array}$ & $\begin{array}{l}49 \% \text { das mulheres apresentaram uma ou mais queixas geniturinárias (que incluem } \\
\text { sintomas do trato urinário, aumento da frequência urinaria e perda do controle da } \\
\text { bexiga),38\% relataram dor na relação sexual e } 45 \% \text { diminuição no interesse sexual; } \\
26 \text { mulheres tiveram filhos após a fratura pélvica, sendo } 38 \% \text { de parto vaginal e } 62 \% \\
\text { cesariana; } 4 \text { das mulheres que tiveram parto vaginal apresentavam fixação cirúrgica da } \\
\text { fratura, demonstrando que mesmo nesses casos, poupando a sínfise púbica, é possível o } \\
\text { parto vaginal. }\end{array}$ \\
\hline
\end{tabular}


(conclusão)

\begin{tabular}{|c|c|c|}
\hline AUTOR & OBJETIVOS & RESULTADOS / CONCLUSÃO \\
\hline $\begin{array}{l}\text { Ramirez, } \\
\text { Lemus, } 2010\end{array}$ & $\begin{array}{l}\text { Determinar a frequência } \\
\text { da disfunção da articulação } \\
\text { sacro-ilíaca mediante o uso } \\
\text { de um grupo de provas } \\
\text { de provocação de dor em } \\
\text { jovens com dor lombar. }\end{array}$ & $\begin{array}{l}\text { Dentre os } 67 \text { participantes, } 24 \text { foram diagnosticados com dor na articulação sacro-ilíaca } \\
\text { (DASI), sendo que } 22 \text { foram mulheres. A frequência de disfunção da articulação sacro- } \\
\text { ilíaca foi de } 35,8 \% \text {. Foi observada associação da disfunção da articulação com o pior } \\
\text { resultado em todos os aspectos avaliados no exame físico. }\end{array}$ \\
\hline $\begin{array}{l}\text { Ares et al., } \\
2012\end{array}$ & $\begin{array}{l}\text { Estudar e descrever as } \\
\text { técnicas de bloqueio e } \\
\text { radiofrequência (RF) na } \\
\text { sacroiliaca. }\end{array}$ & $\begin{array}{l}\text { Os critérios de diagnostico para disfunção sacroilíaca são ambíguos, sendo o bloqueio } \\
\text { com anestésico local o mais aceito. A denervação tem como objetivo conseguir um alivio } \\
\text { da dor mais duradouro que o bloqueio intralocal. As principais técnicas usadas são: RF dos } \\
\text { ramos sacrais laterais, RF fria, RF bipolar e RF usando o dispositivo "Simplicity". }\end{array}$ \\
\hline $\begin{array}{l}\text { Vallier et al., } \\
2012\end{array}$ & $\begin{array}{l}\text { Descrever a função sexual } \\
\text { feminina após lesão da } \\
\text { cintura pélvica. }\end{array}$ & $\begin{array}{l}56 \% \text { das pacientes relataram dispareunia, sendo } 78 \% \text { de paciente com fratura tipo B, } 43 \% \\
\text { com fraturas do tipo C, } 91 \% \text { com lesão por compressão antero-posteior e } 79 \% \text { com fixação } \\
\text { de placas. Fratura sacral ou lesão sacroiliaca com tratamento posterior e mau alinhamento } \\
\text { residual do anel posterior não foram associados com dispareunia. }\end{array}$ \\
\hline $\begin{array}{l}\text { Vleeming et } \\
\text { al., } 2012\end{array}$ & $\begin{array}{l}\text { Estudar a articulação } \\
\text { sacrilíaca, sua anatomia, } \\
\text { função e suas complicações } \\
\text { clinicas. }\end{array}$ & $\begin{array}{l}\text { A dor pélvica causa padrões compensatórios de controle motor a uma frouxidão da } \\
\text { articulação sacroilíaca. Com essa alteração os pacientes têm um fechamento insuficiente } \\
\text { da pelve. O conhecimento desses mecanismos levou ao desenvolvimento de estratégias de } \\
\text { tratamento com estabilização pélvica em posição vertical com o uso de cintos ou fixadores } \\
\text { externos, que simulam uma força de fechamento da sacroilíaca, permitindo que pacientes } \\
\text { com PGP voltem às rotinas diárias. }\end{array}$ \\
\hline
\end{tabular}

Fonte: Dados da pesquisa

a disfunção sacroilíaca em jovens com dor lombar e encontraram, por meio de exame físico, uma alta incidência (35,8\%), sugerindo esta disfunção como uma possível causa de dor lombar.Para os autores, a comprovação causal desta relação poderia facilitar a deteç̧ão e intervenção precoces da lombalgia.

Em um estudo de revisão da anatomia, diagnóstico e tratamento das disfunções sacroilíacas, Mejía et al. (2008) mostraram que o problema possui inúmeras etiologias, apesar de pouco estudadas. Para simplificar, os autores dividiram as origens das disfunções em extra-articular, incluindo como causa mais comum as enteropatias, fraturas, lesões ligamentares e dor miofascial, e intra-articular, como infecções e artrites. Além da etiologia, descreveram fatores que podem predispor dor nesta articulação, como aumento da tensão na articulação - por discrepância no comprimento dos membros inferiores, alteração da marcha, exercício vigoroso prolongado, escoliose e fusão espinhal sacral; aumento na sobrecarga da articulação - por ligamento enfraquecido por violação cirúrgica e hipomobilidade pós-cirúrgica; gravidez - por aumento de peso, alteração postural com hiperlordose, trauma durante o parto e frouxidão ligamentar devido aos níveis hormonais; espondiloartropatias seronegativas; infecção; neoplasia; lesões esportivas; lesão por acidente de carro.

Ainda segundo os autores, além dos exames de imagens como a radiografia, a ressonância magnética e a tomográfica computadorizada, o exame físico também é importante no diagnóstico da disfunção, contando com doze provas provocativas, sugerindo a confirmação do diagnóstico quando três dessas provas são positivas.

Em outro estudo de revisão sobre anatomia, função e complicações clínicas, Vleeming et al. (2012) 
concluíram que modelos de anéis pélvicos propostos para pacientes com dor pélvica podem ser de grande importância no tratamento de estabilização da pelve em posição vertical, permitindo que os pacientes voltem a sua rotina diária. Com o conhecimento dos padróes compensatórios de controle motor, causados pela dor pélvica, a uma frouxidão da articulação, o fechamento da pelve se torna insuficiente e leva ao desenvolvimento de estratégias de tratamento mais eficazes, como os fixadores propostos.

Estudando a relação da disfunção sexual e excretora em homens e mulheres após um ano de fratura pélvica, Wright et al. (2006) descreveram 21\% dos pacientes com alguma disfunção sexual contraposto ao grupo controle, sem fratura, com $14 \%$, além de $8 \%$ de disfunção excretora contra $4 \%$ do grupo controle. Ambos os problemas impactaram negativamente sobre a qualidade de vida, sendo os piores impactos verificados para as lesões sacroilíacas em homens e diástase da sínfise púbica em mulheres.

Também nesse contexto, Vallier (2012) descreveram que $56 \%$ das mulheres com fratura pélvica apresentaram dispareunia- em fraturas do tipo B, tipo C, por compressão antero-posterior e com fixação de placas. Já fratura sacroilíaca com tratamento posterior e mau alinhamento residual do anel posterior não apresentaram relação com disfunção sexual feminina.

Cannada e Barr (2010) estudaram a presença de disfunção sexual em mulheres com fratura pélvica e sua interferência no parto, relatando que 49\% das 66 mulheres estudadas apresentaram uma ou mais queixas geniturinárias, 38\% apresentaram dor na relação sexual e 45\% tiveram diminuição no interesse sexual. Além disso, 26 mulheres tiveram filhos depois da fratura, concluindo que o parto vaginal é possível mesmo para as pacientes submetidas à fixação cirúrgica.

\section{DISCUSSÃO}

A disfunção sexual feminina é um problema global de saúde pública que acomete grande parte da população e prejudica sua qualidade de vida. Sua etiologia é multifatorial e segundo Moreira e Arruda
(2010), alterações dos músculos do assoalho pélvico (MAP) estão entre suas diversas causas.

A relação da anatomia e biomecânica pélvica e a fraqueza da musculatura do assoalho pélvico indicariam que disfunções da articulação sacroilíaca deveriam estar relacionadas à disfunção sexual na mulher. Essa hipótese foi corroborada por três dos estudos encontrados, onde mulheres com tipos diversos de fraturas pélvicas apresentaram concomitantemente disfunção sexual, seja por dor (VALLIER, 2012), dor e disfunção do desejo sexual (CANNADA; BARR, 2010) e dor e disfunções excretoras (WRIGHT et al., 2006). Poucos estudos relacionam diretamente as disfunções sacroilíacas com disfunções sexuais, e os que o fizeram trataram de fraturas da pelve.

No entanto, Costa (2013) estudou a prevalência da disfunção sacroilíaca em portadoras de incontinência urinária e, apesar do número amostral reduzido, encontrou mais de $50 \%$ de correlação, demonstrando ser este mais um aspecto a ser incluído na avaliação global das pacientes com incontinência. Destaca-se ainda que o autor utilizou bases teóricas relacionando a inserção dos músculos do assoalho pélvico (MAP) na região sacral para defender sua hipótese de que alterações posicionais sacroilíacas estariam relacionadas à fraqueza da MAP, tal fraqueza, uma conhecida causa de incontinência urinária.

Sendo incontinência urinária e disfunção sexual problemas pélvicos inter-relacionados (COHEN; BARBOGLIO; GOUSSE, 2008), e estando a incontinência relacionada à disfunção sacroilíaca em geral, é provável que esta última esteja de fato relacionada à disfunção sexual.

Apesar de anormalidades no posicionamento da articulação sacroilíaca, como fixação, hipomobilidade, subluxação ou mau alinhamento, também caracterizarem disfunção desta (RIBEIRO; SCHMIDT; WURFFl, 2003), podendo estas disfunções serem responsáveis pelo aumento na tensão ou fraqueza da musculatura do assoalho pélvico, fundamental na função sexual feminina (BARACHO, 2012), estas bases teóricas não encontraram respaldo na presente revisão. Estudos específicos abordando as disfunções sexuais femininas com distúrbios do alinhamento da articulação sacroilíaca podem ajudar a esclarecer estas questões.

$\mathrm{Na}$ base de dados SciELO, nenhum resultado 
foi encontrado usando as duas expressões juntas, sendo analisados apenas artigos sobre a articulação sacroilíaca em separado, que em sua maioria faziam uma revisão bibliográfica sobre sua anatomia, formas de diagnósticos e tratamentos gerais, não indicando nenhuma informação que relacionava a função sexual, o que dificultou o estudo. Por se tratar de uma base fundamental para a ciência brasileira, de um modo geral, é importante que publicações em língua portuguesa sobre o tema sejam trabalhadas.

Considerando a prevalência importante de disfunções sacroilíacas na população em geral, bem como o aspecto multicausal da disfunção sexual, o aprofundamento de estudos que esclareçam esta relação pode trazer benefícios imensuráveis tanto no campo da prevenção quanto no tratamento destes problemas, que impactam negativamente sobre a qualidade de vida.

\section{CONCLUSÃO}

Fraturas da cintura pélvica estão relacionadas à disfunção sexual feminina, Apesar de as bases teóricas apontarem clara relação entre outras disfunções sacroilíacas que não fraturas e a disfunção sexual, esta relação não teve respaldo na literatura. Urgem estudos explorando as alterações anatômicas e biomecânicas da articulação sacroilíaca, especialmente as que gerem fraqueza ou tensão da musculatura do assoalho pélvico como possíveis fatores etiológicos da disfunção sexual.

\section{REFERÊNCIAS}

ABDO, C. H. N.; OLIVEIRA, J. R. W. M.; MOREIRA, E. D.Perfil sexual da população brasileira: resultados do Estudo do Comportamento Sexual (ECOS) do Brasileiro. Rev Bras Med. v.59, n. 4, p.250-257, 2002.

ABDO, C. H. N. Descobrimento sexual do Brasil. São Paulo: Summus, 2004.

ABDO, C. H. N. Quociente sexual feminino: um questionário brasileiro para avaliar a atividade sexual da mulher. Diagn Tratamento, v.14, n. 2, p. 89-91, 2009.
ARES, J. A.; AMATRIAÍN, G. R.; GONZÁLEZ, A. P.; Nieto, C.; López, D. Bloqueo y radiofrecuencia de la articulación sacroilíaca. Rev. Soc. Esp., Dolor, v. 19, n. 6, 2012.

BARACHO, E. Fisioterapia aplicada à saúde da mulher. 5. ed. Rio de Janeiro: Guanabara Koogan, 2012.

BASSON, R.; ALTHOF, S.; DAVIS S.; FUGL-MEYER, K.; GOLDSTEIN, I.; LEIBLUM, S.; MESTON, C.; ROSEN, R.; WAGNER, G. Summary of the Recommendations on Sexual Dysfunctions in Women. J Sex Med. , v. 1, n. 1, 2004.

CALAIS-GERMAIN, B. O períneo feminino e o parto: elementos de Anatomia e Exercícios Práticos. Barueri: Manole, 2005.

CANNADA. L. K.; BARR, J. Pelvic Fractures in Women of Childbearing Age. Clin Orthop Relat Res., v. 468, n. 7, p. 1781-1789, 2010.

COHEN, B.L.; BARBOGLIO, P.; GOUSSE, A. The impact of lower urinary tract symptoms and urinary incontinence on female sexual dysfunction using a validated instrument. J Sex Med., v.5, n. 6, p.1418-1423, 2008.

FORTUNATO, G. L., ALIBERTE P. I., ANGELIN E. C. N., GRUBER C. R.Correlação entre a força dos músculos do assoalho pélvico e a satisfação sexual de mulheres. Cadernos da Escola de Saúde, Curitiba, v.2, p.143-158, 2006.

GIRALDO, P.C.; POLPETA, N.C.; JULIATO, C.R.T.; YOSHIDA, L. P.; ELEUTÉRIO JUNIOR, J. Evaluation of Sexual Function in Brazilian Women with Recurrent Vulvovaginal Candidiasis and Localized Provoked Vulvodynia. J Sex Med., 2012.

HENTSCHEL, H.; ALBERTON, D. L.; CAPP, E.; GOLDIM, J. R.; PASSOS, E. P. Aspectos Fisiológicos e Disfuncionais da Sexualidade Feminina. Rev HCPA., v.26, n. 2, p. 61-65, 2006.

JONES, L. R. The use of validated questionnaires to assess female sexual dysfunction. World J Urol., v.20, n. 2, p.89$92,2002$. 
LARA, L.A.S.; SILVA, A.C.J.S.R.; ROMÃO, A.P.M.S.; JUNQUEIRA, F.R.R.Abordagem das disfunções sexuais femininas. Rev. Bras. Ginecol. Obstet., v.30, n.6, 2008.

MAGEE, D.J. Avaliação músculo esquelética. 4. ed. Barueri: Manole, 2005

MEJIA, G. E.; ARIAS, M.; VALDEZ, K.; CARRILLO, S.; Infante, G. Dolor de la articulación sacroilíaca: Anatomía, Diagnóstico y Tratamiento.Rev. Soc. Esp., Dolor, v. 15, n. 3, 2008 .

MIRANDA, R.; SCHOR, E.; GIRÃO, M. J. B. C. Avaliação postural em mulheres com dor pélvica crônica. Rev. Bras. Ginecol. Obstet., v.31, n. 7, p.353-360, 2009.

MOREIRA, E. C. H., ARRUDA, P. B. Força Muscular do Assoalho Pélvico entre Mulheres Continentes Jovens e Climatéricas. Semina: Ciências Biológicas da Saúde, Londrina, v. 31, n. 1, p. 53-61, 2010.

PIASSAROLLI, V. P.; HARDY, E.; ANDRADE, N. F. et al. Treinamento dos músculos do assoalho pélvico nas disfunções sexuais femininas. Rev. Bras. Ginecol. Obstet., v.32, n. 5, p.234-240, 2010.

RAMIREZ, C.; LEMUS, D. M. C. Disfunção da articulação sacro-ilíaca em jovens com dor lombar. Fisioterapia em movimento, v. 23, n. 3, 2010.

RIBEIRO, S.; SCHMIDT, A. P.; WURFF, P. V. D. Disfunção sacroilíaca. Acta ortop. Bras., v. 11, n. 2, p. 118-125, 2003.

SOARES, G. M. M.; ARCANJO, G. N.; PIRES, J. L. V. Disfunção osteopática pélvica em mulheres com incontinência urinária de esforço. Rev Terapia Manual, v.9, n. 45, p. 534-540, out de 2011

VALLIER, H. A.; CURETON, B. A.; SCHUBECK, D. Pelvic ring injury is associated with sexual dysfunction in women. J Orthop Trauma, v.26, n. 5, p. 308-313, 2012.

VLEEMING, A.; SCHUENKE, M. D., MASI, A. T.; CARREIRO, J.E.; DANNEELS, L.; WILLARD, F.H. The sacroiliac joint: an overview of its anatomy, function and potential clinical implications. Journal of Anatomy, v. 221, n. 6, p.537$567,2012$.
YENG, L.T.; TEIXEIRA, M.J.; UNGARETTI Jr, et al. Dor pelviperineal. Rev. Med., v.80, n. ed. esp.2, p.351-374, 2001.

WRIGHT, J. L.; NATHENS, A. B.; RIVARA, F. P. MacKenzie, E. J.; Wessells, H. Specific fracture configurations predict sexual and excretory dysfunction in men and women 1 year after pelvic fracture. J Urolog y., v.176, n. 4, p.1540$1545,2006$.

Recebido em: 01 setembro de 2016 Aceito em: 04 dezembro de 2016 